\title{
Intervención médica invasiva comparada con la intervención conservadora en el tratamiento de varicosidades en mujeres gestantes: Evaluación rápida de la evidencia ${ }^{1}$
}

\author{
Pilar Cardenas Sánchez², Jennifer González Bermúdez ${ }^{3}$
}

Institución: Universidad de Costa Rica

\section{RESUMEN}

El objetivo de este estudio fue analizar la mejor evidencia científica disponible relacionada con la intervención médica conservadora comparada con la intervención más invasiva incluyendo la quirúrgica para el tratamiento de venas varicosas en miembros inferiores durante el embarazo y la prevención de la aparición posterior. Inició con Se aplicó la metodología de evaluación rápida de la evidencia. Se llevó a cabo una búsqueda de literatura en las bases de datos, sin límite de fechas. Criterios de inclusión: Gestantes con patología varicosa en miembros inferiores de cualquier edad. Los tipos de estudios analizados fueron Revisiones Sistemáticas, ECA, Meta-análisis o Guías de Práctica Clínica en inglés, portugués o español. Se utilizó la plataforma FLC 2.0 y Agree II para el análisis y la plantilla de Sackett para la interpretación de niveles de evidencia y grados de recomendación. Se recuperaron 322 artículos, se seleccionaron tres. Se obtuvo que la intervención recomendada es la terapia conservadora, solo en casos excepcionales se determinará otra intervención. El tratamiento invasivo incluyendo el quirúrgico, se reserva para casos delicados ya que los riesgos superan los beneficios. Se concluye que el tratamiento de primera línea para el tratamiento de varicosidades en gestantes son las medias de compresión. Los rutósidos y anticoagulantes orales parecen ayudar pero no es seguro de utilizar durante el embarazo.

Palabras clave: Embarazo; Várices; Tratamiento-conservador; Tratamiento-farmacológico.

DOI 10.15517/revenf.v0i38.37593

${ }^{1}$ Fecha de recepción: 06 de junio del 2019

${ }^{2}$ Enfermera, Máster en Ginecología, Obstetricia y Perinatología. Docente de la Universidad de Costa Rica. Costa Rica. Correo electrónico: pili.cardenas28@gmail.com

${ }^{3}$ Enfermera, Máster en Ginecología, Obstetricia y Perinatología. Trabajo independiente. Costa Rica. Correo electrónico: jenigo78@gmail.com 


\title{
Invasive medical intervention compared with conservative intervention in the treatment of varicose veins in pregnant women: Rapid evaluation of the evidence ${ }^{1}$
}

Institution: University of Costa Rica

\author{
Pilar Cardenas Sánchez², Jennifer González Bermúdez ${ }^{3}$
}

\begin{abstract}
The objective of this study was to analyze the best available scientific evidence related to conservative medical intervention compared to the most invasive intervention including surgery for the treatment of varicose veins in the lower limbs during pregnancy and the prevention of subsequent onset. It started with The rapid evidence evaluation methodology was applied. A search of literature in the databases was carried out, with no date limit. Inclusion criteria: Pregnant women with varicose pathology in lower limbs of any age. The types of studies analyzed were Systematic Reviews, RCTs, Meta-analysis or Clinical Practice Guidelines in English, Portuguese or Spanish. The FLC 2.0 and Agree II platform was used for the analysis and the Sackett template for the interpretation of levels of evidence and grades of recommendation. 322 items were retrieved, three were selected. It was obtained that the recommended intervention is conservative therapy, only in exceptional cases will another intervention be determined. Invasive treatment, including surgical treatment, is reserved for sensitive cases since the risks outweigh the benefits. It is concluded that the first line treatment for the treatment of varicose veins in pregnant women is compression stockings. Routine and oral anticoagulants seem to help but it is not safe to use during pregnancy.
\end{abstract}

Keywords: Pregnancy; Varicose-veins; Conservative-treatment; Pharmacological-treatment.

DOI 10.15517/revenf.v0i38.37593

${ }^{1}$ Date of receipt: June 6, 2019

Date of acceptance: November 6, 2019

${ }^{2}$ Nurse, Master in Gynecology, Obstetrics and Perinatology. Professor of the University of Costa Rica. Costa Rica. E-mail: pili.cardenas28@gmail.com

${ }^{3}$ Nurse, Master in Gynecology, Obstetrics and Perinatology. Independent work. Costa Rica. E-mail: jenigo78@gmail.com 


\title{
Intervenção médica invasiva em comparação com intervenção conservadora no tratamento de varizes em mulheres grávidas: avaliação rápida da evidência ${ }^{1}$
}

Instituição: Universidade da Costa Rica

Pilar Cardenas Sánchez², Jennifer González Bermúdez ${ }^{3}$

\begin{abstract}
RESUMO
O objetivo deste estudo foi analisar as melhores evidências científicas disponíveis relacionadas à intervenção médica conservadora em comparação com a intervenção mais invasiva, incluindo cirurgia para o tratamento de varizes nos membros inferiores durante a gravidez e a prevenção de subsequente aparecimento. Começou com A metodologia de avaliação rápida de evidências foi aplicada. Foi realizada uma pesquisa bibliográfica nas bases de dados, sem data limite. Critérios de inclusão: Gestantes com patologia varicosa em membros inferiores de qualquer idade. Os tipos de estudos analisados foram Revisões Sistemáticas, ECR, Meta-análise ou Diretrizes de Prática Clínica em inglês, português ou espanhol. A plataforma FLC 2.0 e Agree II foi utilizada para a análise e o modelo de Sackett para a interpretação dos níveis de evidência e graus de recomendação. 322 itens foram recuperados, três foram selecionados. Foi obtido que a intervenção recomendada é terapia conservadora, apenas em casos excepcionais será determinada outra intervenção. O tratamento invasivo, incluindo o tratamento cirúrgico, é reservado para casos sensíveis, pois os riscos superam os benefícios. Conclui-se que o tratamento de primeira linha para o tratamento de varizes em gestantes são as meias de compressão. Anticoagulantes de rotina e orais parecem ajudar, mas não é seguro para uso durante a gravidez.
\end{abstract}

Palavras-chave: Gravidez; Varizes; Tratamento-conservador; Tratamento-farmacológico.

DOI 10.15517/revenf.v0i38.37593

${ }^{1}$ Data de recebimento: 6 de junho de 2019

Data de aceitação: 26 de novembro del 2019

${ }^{2}$ Enfermeira. Mestre em Ginecologia, Obstetrícia e Perinatologia. Professor da Universidade da Costa Rica. Costa Rica. Correio eletrônico: pili.cardenas28@gmail.com

${ }^{3}$ Enfermeira. Mestre em Ginecologia, Obstetrícia e Perinatologia. Trabalho independente. Costa Rica. Correio eletrônico: jenigo78@gmail.com 


\section{INTRODUCCIÓN}

La evidencia científica señala que el proceso de embarazo viene acompañado de múltiples cambios tanto hormonales como fisiológicos. Dichos cambios aunados con los hemostáticos pueden desencadenar problemas de salud o molestias durante la gestación. Dentro de este contexto, ejemplo de la problemática, es la prevalencia y la incidencia de venas varicosas en el embarazo que pueden conducir a otras complicaciones.

Alrededor del $70-80 \%$ de las gestantes tienen altas probabilidades de desarrollar varicosidades que pueden dar inicio desde el primer trimestre de embarazo, acompañadas de una incómoda sintomatología, factores de riesgo y calidad de vida alterado.

Cossart ${ }^{1}$ en el 2001, realizó un estudio titulado "Venas varicosas y embarazo"; el autor señala que es común que dicho problema de salud se presente durante este periodo, además menciona que una mínima dilatación venosa conlleva a enfocarse a un actuar conservador; pero si se presentan venas más dilatadas se debe realizar a un tratamiento más intervencionista.

En este mismo año, Thaler, Huch y $\mathrm{Huch}^{2}$ realizaron un estudio en el Hospital Universitario de Zurich, Suiza, donde determinaron que las medias suficientemente compresivas (mayor a $32 \mathrm{mmHg}$ ) mejoran el retorno venoso de las piernas durante el embarazo y, por lo tanto, pueden prevenir la trombosis en pacientes de alto riesgo y mejoran la sintomatología.

En el año 2006, Bamigboye y Hofmeyr ${ }^{3}$, publicaron un estudio en el que se concluyó que los rutósidos aparentan aliviar los síntomas de la insuficiencia venosa y embarazos avanzados; sin embargo, no se conoce si esta droga es segura en la gestación. Por otro lado, se expone que la compresión neumática externa reduce los síntomas pero no las venas varicosas.

Por su parte, en el 2009, Lenkovic, Cabrijan. Gruber y otros ${ }^{4}$, realizaron un estudio sobre la contribución de la progesterona en el desarrollo de venas varicosas en miembros inferiores durante el embarazo, donde se demostró que el promedio de concentración de progesterona en el grupo de estudio con venas dilatadas fue mayor que en el grupo control. Estos hallazgos apoyan el rol del factor hormonal en el desarrollo de venas varicosas en mujeres.

Por otro lado, en el año 2014, Sell, Vikatmaa, Albäck, Lepäntalo, y otros ${ }^{5}$ realizaron un estudio sobre la terapia de comprensión vs cirugía, donde se concluyó que la eliminación quirúrgica del reflujo venoso superficial no complicado es un tratamiento eficaz en comparación con sólo proporcionar las medias de compresión.

Referente al tema de estudio de la investigación, es importante mencionar que las venas varicosas se presentan con mucha frecuencia en las mujeres gestantes. Según las estadísticas, la manifestación ronda entre el $20 \%$ de las primíparas, $30 \%$ de las secundíparas y hasta el $57 \%$ de las multíparas ${ }^{6}$.

A nivel económico, Vandy et al. ${ }^{7}$ y Zhang et al. ${ }^{8}$, señalan que este padecimiento asociado a la presencia de úlceras venosas -en los Estados Unidos- excede en el gasto en US\$1 billón anualmente, estas pérdidas económicas influyen también a nivel laboral cuando incurren en incapacidades. Según Jundt, Liem y Moneta ${ }^{9}$ el $100 \%$ de las personas con insuficiencia venosa crónica experimentan un impacto negativo en su trabajo, el cual se estima para 
el país en dos millones de días de trabajo perdidos por año. Por otro lado, Lohr et al. ${ }^{6}$, mencionan que a medida que el proceso de enfermedad venosa avanza la cantidad de cuidados necesarios y los costos aumentan.

Entre los síntomas reportados por las gestantes están: dolor, sensación de pesadez de los miembros inferiores, picazón, hinchazón, calambres, hormigueo, cambios en la coloración de la piel, edema, entre otros ${ }^{6,7,10}$. En cuanto a los factores de riesgo reportados en la formación de venas varicosas están: embarazo, número de embarazos, la edad, obesidad, mucho tiempo en una misma posición y sexo femenino ${ }^{6,11,12,13,14}$. Partiendo de este contexto, es importante que las usuarias sean educadas acerca de los cambios en el estilo de vida y las diferentes opciones de tratamiento con las que pueden contar ${ }^{6}$, ya sea el tratamiento farmacológico, no farmacológico o el quirúrgico.

Lo anterior es razón por la cual los profesionales en Gineco-Obstetricia deben conocer y entender desde una base científica las diferentes opciones de tratamiento que existen para personas que presentan dicha patología. Es por ello que el objetivo del estudio fué analizar la mejor evidencia científica disponible relacionada con la intervención médica conservadora comparada con la intervención más invasiva incluyendo la quirúrgica para el tratamiento de venas varicosas en miembros inferiores durante el embarazo y la prevención de la aparición posterior.

Esta investigación tiene implicaciones para la práctica prenatal y postnatal de la Enfermería Gineco-Obstétrica, dado que incide en una práctica actualizada y basada en la mejor evidencia científica disponible para la toma de decisiones calificada en la atención de la mujer embarazada.

\section{MATERIALES Y MÉTODO}

El presente estudio corresponde a una investigación aplicada de tipo secundaria, la cual es entendida como la utilización de los conocimientos en la práctica para el enriquecimiento de la disciplina ${ }^{15}$, para luego implementar y sistematizar la práctica basada en investigación ${ }^{16}$.

Actualmente, se puede considerar la Enfermería Basada en la Evidencia como una estrategia en donde la investigación apoya el cambio de pensamientos y prácticas en el quehacer de la disciplina, esto por medio de la mejor evidencia científica ${ }^{17,18}$.

Los estudios secundarios brindan un gran soporte, ya que devienen de un estudio previo, que realiza revisiones exhaustivas de otras investigaciones ${ }^{19}$ transformándola en una herramienta confiable para la aplicación de la Práctica de Enfermería Basada en la Evidencia. A continuación, se presentan los aspectos metodológicos que guiaron este estudio y que consta de cinco pasos o fases.

\section{Fase 0: Spirit of inquiry.}

Corresponde al surgimiento de una inquietud que conduce a interrogarse acerca del porqué de los cuidados prestados a los usuarios en su realidad. En la presente investigación surge la inquietud al observar desconocimiento y consultar con profesionales en Enfermería Gineco- Obstetricia sobre el abordaje sin fundamento científico en la atención, tratamiento y prevención de las venas varicosas en mujeres gestantes.

\section{Fase 1: Formulación de la Pregunta Clínica.}


La pregunta clínica requiere de un enfoque sistematizado para ser respondida, por lo cual utiliza el acrónimo PICO (población, intervención, comparación y resultados).

Para el caso de este estudio la pregunta planteada es la siguiente: ¿En mujeres gestantes, la intervención médica conservadora comparada con la intervención invasiva incluyendo la quirúrgica, es más efectiva para el tratamiento y prevención de la aparición posterior de las venas varicosas en miembros inferiores?

Una vez establecido el tipo de pregunta de la investigación se determinaron los tipos de estudio más idóneos que respondieron a la pregunta elaborada, los cuales son: Ensayos Clínicos controlados aleatorizados (ECAS), Revisiones Sistemáticas, Meta-análisis y Guías de Prácticas Clínicas

\section{Fase 2: Búsqueda de la evidencia científica.}

Para desarrollar este paso se establece una línea de trabajo por medio de: Estrategia de búsqueda y establecimiento de la pregunta PICO.

\section{Tabla 1. Descripción de la Pregunta clínica según acrónimo PICO.}

\begin{tabular}{|c|c|c|c|}
\hline $\mathbf{P}$ & I & $\mathbf{C}$ & $\mathbf{O}$ \\
\hline Problema de interés & $\begin{array}{l}\text { Intervención que se va a } \\
\text { considerar }\end{array}$ & $\begin{array}{l}\text { Intervención con la que se } \\
\text { va a comparar }\end{array}$ & $\begin{array}{l}\text { Resultado clínico que se } \\
\text { valora }\end{array}$ \\
\hline $\begin{array}{l}\text { Mujeres gestantes con } \\
\text { venas varicosas }\end{array}$ & $\begin{array}{l}\text { Tratamiento médico } \\
\text { conservador para el } \\
\text { tratamiento de las varices } \\
\text { en miembros inferiores }\end{array}$ & $\begin{array}{l}\text { Tratamiento médico } \\
\text { invasivo, incluyendo el } \\
\text { Quirúrgico para el } \\
\text { tratamiento de varices en } \\
\text { MI }\end{array}$ & $\begin{array}{l}\text { Efectividad } \\
\text { tratamiento del } \\
\text { prevención de varices en } \\
\text { MI y aparición posterior. }\end{array}$ \\
\hline
\end{tabular}

Fuente: Elaboración propia.

\section{Mapa Estratégico:}

- Determinación de descriptores específicos: Se establecieron a partir de la pregunta clínica en idioma español, inglés y portugués los cuales fueron: Embarazo, Venas Varicosas, Tratamiento, Extremidades inferiores, Tratamiento Farmacológico, Tratamiento Conservador y Tratamiento Quirúrgico.

- Relación entre conceptos formulados: Embarazo con venas varicosas, venas varicosas en extremidades inferiores, tratamiento para venas varicosas, extremidades inferiores con tratamiento para venas varicosas, tratamiento farmacológico para venas varicosas, tratamiento conservador para venas varicosas, tratamiento quirúrgico para venas varicosas.

- Traducción de descriptores: La mayor parte de la evidencia científica de alta calidad se encuentra en el idioma inglés y portugués, por lo tanto, la traducción de descriptores es necesaria para la obtención de la evidencia en la bases de datos.

- Criterios de inclusión para la recuperación de la evidencia (Tabla 2)

Tabla 2. Descripción de los criterios de inclusión, 2017. 


\begin{tabular}{|l|l|}
\hline \multicolumn{2}{|c|}{ Criterios de inclusión } \\
\hline Tiempo & No hubo restricción de tiempo, la búsqueda incluyo artículos desde 1939 hasta el año 2017. \\
\hline Población & Mujeres embarazadas con enfermedad varicosa. \\
\hline Tipo de estudio & Revisiones Sistemáticas, Guías Prácticas Clínicas, ECAS y Meta-análisis. \\
\hline Idioma & Publicaciones en español, inglés y portugués. \\
\hline
\end{tabular}

Fuente: Elaboración propia

Sitios de búsqueda utilizados para el estudio: PubMed, Science Direct, Cochrane Library, Scielo y Google Académico.

\section{Establecimiento del algoritmo de búsqueda.}

Está diseñado para localizar un elemento con ciertas propiedades dentro de una estructura o base de datos. El algoritmo de búsqueda da como resultado un número de artículos que posteriormente deberán ser objeto de lectura crítica. (Figura 1)

Figura 1. Presentación del algoritmo de búsqueda, 2017.

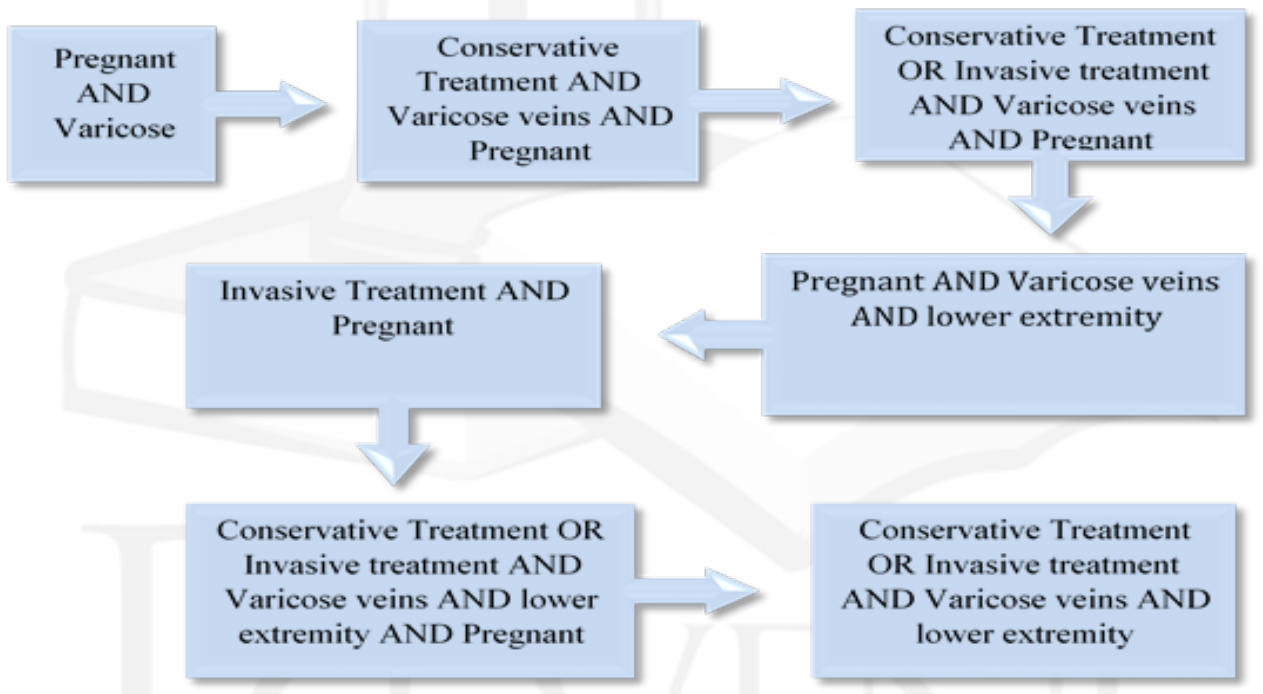

Fuente: Elaboración propia

\section{Fase 3: Valoración crítica de la evidencia.}

Se trabajó con diferentes tipos de instrumentos que permitieron validar la información. Se utilizó FLC 2.0 (ficha de lectura crítica), que es una herramienta que permite analizar la calidad de las pruebas científicas y generar tablas de evidencia. En cuanto a las Guías de Práctica Clínica (GPC), se utilizó el instrumento AGREE II, última versión. Según Flores y Montoya ${ }^{20}$, es un instrumento desarrollado para realizar una evaluación crítica de las guías de práctica clínica.

Finalmente, para la interpretación de los niveles de evidencia y grados de recomendación se utilizó el instrumento propuesto por Sackett ${ }^{21}$ muy recomendada para analizar críticamente estudios de terapia o intervención. 


\section{Fase 4: Transferencia de la Evidencia a la Práctica Clínica.}

La transferencia de la evidencia se realiza con el objetivo de implementar cambios en la práctica clínica (si es considerado necesario), por lo cual es importante compartir dichos resultados con los profesionales en salud e instituciones sanitarias.

Referente a esta investigación, se tomó la decisión de llevar a cabo la difusión de los resultados obtenidos por medio de un boletín informativo que contiene un resumen de la investigación y las conclusiones obtenidas.

Se procedió a visitar cada unidad de maternidad y entregar un total de 50 boletines por hospital. La información impresa (boletines) se entregó al personal sin costo alguno. En total se visitaron nueve centros hospitalarios: Hospital Adolfo Carit Eva, Hospital México, Hospital San Juan de Dios, Hospital Dr. Rafael Ángel Calderón Guardia, Hospital San Rafael de Alajuela, Hospital Max Peralta de Cartago, Hospital de Ciudad Neily, Hospital de la Anexión en Nicoya y Hospital Enrique Baltodano Briceño en Liberia.

Se realizó la exposición de la presente investigación al personal de Enfermería Gineco-Obstétrica del Hospital Adolfo Carit Eva. También se realizó la publicación de dos artículos científicos a través de la Revista Científica de la Escuela de Enfermería, con el fin de propiciar una divulgación más amplia de los resultados de la investigación.

\section{Fase 5: Evaluación de los resultados de la utilización de la evidencia.}

En relación a esta fase y lo que corresponde a esta investigación, después de la transferencia de resultados e implementación de las nuevas prácticas en instituciones de salud, la evaluación quedará a cargo de los profesionales en salud.

Para fines de nuestro estudio la evaluación de la transferencia de la información está dada por el número de enfermeras que presenciaron la presentación del estudio, el número de folletos informativos distribuidos en las diferentes instituciones de salud y por la publicación de dos artículos científicos.

\section{Consideraciones Éticas.}

Según Laguna, Caballero, Lewis, Mazueral y otros $^{23}$, mencionan que, el plagio es un apropiamiento de pensamiento intelectual de alguien más o la copia de resultados de investigaciones hechas por otros sin autorización. En el estudio realizado, los artículos y documentos científicos citados se efectuaron conforme se establece en Vancouver. En relación con el conflicto de intereses, éste se describe como: cuando un autor tiene relaciones personales o financieras que puedan influenciar las acciones tomadas. En el caso de este estudio no se presentaron conflictos de intereses por parte de las investigadoras. También se tomó en cuenta la confidencialidad y privacidad, que se refiere a que los usuarios tienen derecho a la privacidad, su información personal u hospitalaria no debe ser publicada, a menos que el usuario otorgue un consentimiento informado.

\section{RESULTADOS}


En la búsqueda se recuperó un total de 322 artículos, mismos que fueron sometidos a una serie de pasos para descartar los artículos que no respondían a la pregunta clínica.

Gran parte de los resultados fueron obtenidos con las relaciones: Conservative treatment or invasive treatment and varicose veins and lower extremity (136), Pregnant and varicose veins (60), Invasive treatment and pregnant (48) y Conservative treatment or invasive treatment and varicose veins and lower extremity and pregnant (42).

A continuación, se presenta el resumen del procedimiento de selección de estudios, y las bases de datos en las que se repiten.

Tabla 3. Resumen del proceso de selección por base de datos, 2017.

\begin{tabular}{|l|c|c|c|c|c|c|}
\hline \multirow{2}{*}{ PROCESO DE SELECCIÓN } & \multicolumn{7}{|c|}{ BASES DE DATOS } \\
\cline { 2 - 7 } & Pubmed & Cochrane & $\begin{array}{c}\text { Sciencie } \\
\text { Direct }\end{array}$ & Scielo & $\begin{array}{c}\text { Otras } \\
\text { Fuentes }\end{array}$ & TOTAL \\
\hline Titulo & 19 & 4 & 12 & 42 & 12 & 89 \\
\hline Abstract & 15 & 8 & 10 & 39 & 41 & 113 \\
\hline Duplicados & 47 & 5 & 13 & 8 & 7 & 80 \\
\hline No texto completo & 4 & 6 & 19 & 6 & 2 & 37 \\
\hline $\begin{array}{l}\text { Seleccionados para análisis } \\
\text { crítico }\end{array}$ & 1 & 1 & 1 & 0 & 0 & 3 \\
\hline TOTAL & 86 & 24 & 55 & 95 & 62 & 322 \\
\hline
\end{tabular}

Fuente: Elaboración Propia.

En las tablas 4 y 5 se visualiza el procesamiento de información de los 3 artículos seleccionados e incluidos para el análisis crítico, además del nivel de evidencia según el programa FLC 2.0 y AGREE II. 
Tabla 4. Inclusión de los artículos para análisis crítico, 2017.

\begin{tabular}{|c|c|c|c|c|c|c|c|c|}
\hline Referencia & Estudio & Población & Intervención & Comparación & Resultados & Conclusión & Comentario & $\begin{array}{l}\text { Calidad de } \\
\text { la evidencia }\end{array}$ \\
\hline $\begin{array}{l}\text { Cita } \\
\text { abreviada: } \\
\text { Taylor } \\
2017\end{array}$ & $\begin{array}{l}\text { Objetivos: } \\
\text { Destacar los } \\
\text { cambios } \\
\text { fisiológicos y } \\
\text { hemodinámicos } \\
\text { específicos que } \\
\text { ocurren durante } \\
\text { el embarazo y } \\
\text { examinar las } \\
\text { intervenciones } \\
\text { no } \\
\text { farmacológicas, } \\
\text { farmacológicas } \\
\text { e invasivas que } \\
\text { son apropiadas } \\
\text { para la } \\
\text { profilaxis y el } \\
\text { tratamiento del } \\
\text { Insuficiencia } \\
\text { Venosa Crónica } \\
\text { Primaria y } \\
\text { Secundaria y } \\
\text { del } \\
\text { tromboembolis } \\
\text { mo venoso. } \\
\text { Periodo de } \\
\text { búsqueda: } \\
\text { 2017 } \\
\text { Diseño: } \\
\text { Revisión } \\
\text { Sistemática de } \\
\text { la literatura } \\
\text { relacionada con } \\
\text { el TEV y el IVC } \\
\text { en el embarazo. }\end{array}$ & $\begin{array}{l}\text { Población: } \\
\text { En el } \\
\text { estudio se } \\
\text { define } \\
\text { claramente } \\
\text { que las } \\
\text { mujeres } \\
\text { gestantes } \\
\text { son la } \\
\text { población } \\
\text { objeto de } \\
\text { estudio }\end{array}$ & $\begin{array}{l}\text { Intervención: } \\
\text { Este artículo de } \\
\text { revisión destaca } \\
\text { los cambios } \\
\text { fisiológicos y } \\
\text { hemodinámicos } \\
\text { específicos que } \\
\text { ocurren durante } \\
\text { el embarazo y } \\
\text { examina las } \\
\text { intervenciones } \\
\text { no } \\
\text { farmacológicas, } \\
\text { farmacológicas } \\
\text { e invasivas que } \\
\text { son apropiadas } \\
\text { para la } \\
\text { profilaxis y el } \\
\text { tratamiento del } \\
\text { IVC y del } \\
\text { tromboembolis } \\
\text { mo venoso } \\
\text { (TEV). }\end{array}$ & $\begin{array}{l}\text { Comparación: } \\
\text { Se comparan las } \\
\text { intervenciones } \\
\text { no } \\
\text { farmacológicas } \\
\text { y } \\
\text { farmacológicas } \\
\text { o invasivas en } \\
\text { el tratamiento } \\
\text { de las venas } \\
\text { varicosas en } \\
\text { miembros } \\
\text { inferiores de la } \\
\text { mujer gestante }\end{array}$ & $\begin{array}{l}\mathbf{N}^{0} \text { de estudios y } \\
\text { pacientes: } \\
\text { Parcialmente } \\
\text { Magnitud del efecto: } \\
\text { Como resultado, faltan } \\
\text { ensayos controlados } \\
\text { aleatorios grandes, } \\
\text { prospectivos y escasean } \\
\text { los datos con respecto a } \\
\text { los resultados a largo } \\
\text { plazo tanto para las } \\
\text { mujeres como para sus } \\
\text { bebés como resultado de } \\
\text { las intervenciones } \\
\text { farmacológicas que se } \\
\text { utilizan habitualmente en } \\
\text { mujeres no embarazadas } \\
\text { con IVC crónica } \\
\text { primaria y secundaria. } \\
\text { Por otro lado los } \\
\text { rutósidos son la } \\
\text { intervención más } \\
\text { efectiva para reducir los } \\
\text { síntomas de las venas } \\
\text { varicosas durante el } \\
\text { embarazo. Sin embargo, } \\
\text { este hallazgo se basa en } \\
\text { un único estudio de } \\
\text { 1975, con datos } \\
\text { limitados sobre la } \\
\text { seguridad del } \\
\text { medicamento durante el } \\
\text { embarazo. Se descubrió } \\
\text { que tanto la reflexología } \\
\text { como la inmersión en } \\
\text { agua reducen los } \\
\text { síntomas del edema de la } \\
\text { pierna; sin embargo, } \\
\text { estos hallazgos se } \\
\text { basaron en estudios } \\
\text { únicos que no se han } \\
\text { reproducido. Las prendas } \\
\text { de compresión también } \\
\text { pueden ser útiles para el } \\
\text { control de los síntomas y } \\
\text { son una herramienta } \\
\text { económica que se puede }\end{array}$ & $\begin{array}{l}\text { Conclusiones } \\
\text { : } \\
\text { El embarazo } \\
\text { tiene efectos } \\
\text { significativos } \\
\text { sobre el } \\
\text { sistema } \\
\text { venoso de las } \\
\text { extremidades } \\
\text { inferiores. El } \\
\text { aumento de } \\
\text { los diámetros } \\
\text { venosos y del } \\
\text { volumen de } \\
\text { sangre, en } \\
\text { combinación } \\
\text { con un flujo } \\
\text { reducido } \\
\text { dentro de las } \\
\text { venas } \\
\text { profundas, } \\
\text { predispone a } \\
\text { las mujeres } \\
\text { embarazadas } \\
\text { a IVC } \\
\text { primario y } \\
\text { secundario. } \\
\text { La profilaxis } \\
\text { y el } \\
\text { tratamiento de } \\
\text { TEV se han } \\
\text { estudiado } \\
\text { extensamente } \\
\text { en el } \\
\text { embarazo, y } \\
\text { las directrices } \\
\text { de gestión } \\
\text { han sido } \\
\text { publicadas } \\
\text { por } \\
\text { organizacione } \\
\text { s } \\
\text { profesionales, } \\
\text { incluido el } \\
\text { Colegio } \\
\text { Estadouniden } \\
\text { se de } \\
\text { Obstetras y } \\
\text { Ginecólogos. }\end{array}$ & $\begin{array}{l}\text { Comentarios: } \\
\text { Los cambios } \\
\text { fisiológicos en } \\
\text { todo el sistema } \\
\text { arterial y venoso } \\
\text { durante el } \\
\text { embarazo están } \\
\text { bien } \\
\text { documentados. } \\
\text { Sin embargo, hay } \\
\text { una escasez de } \\
\text { datos disponibles } \\
\text { para construir } \\
\text { pautas de } \\
\text { atención, } \\
\text { particularmente } \\
\text { en la paciente } \\
\text { embarazada con } \\
\text { venas varicosas } \\
\text { que podrían } \\
\text { progresar a una } \\
\text { insuficiencia } \\
\text { venosa superficial } \\
\text { sintomática. Se } \\
\text { requiere una } \\
\text { investigación } \\
\text { adicional en } \\
\text { forma de ensayos } \\
\text { aleatorios } \\
\text { prospectivos para } \\
\text { establecer pautas } \\
\text { apropiadas para el } \\
\text { tratamiento. }\end{array}$ & $\begin{array}{l}\text { Calidad de } \\
\text { la } \\
\text { evidencia: } \\
\text { Media }\end{array}$ \\
\hline
\end{tabular}




\begin{tabular}{|c|c|c|c|c|c|c|c|c|}
\hline Referencia & Estudio & Población & Intervención & Comparación & Resultados & Conclusión & Comentario & $\begin{array}{l}\text { Calidad de } \\
\text { la evidencia }\end{array}$ \\
\hline & & & & & $\begin{array}{l}\text { aplicar fácilmente. El } \\
\text { riesgo relativo de } \\
\text { aparición de TEV } \\
\text { durante el embarazo es } \\
\text { elevado, aunque la el } \\
\text { riesgo absoluto sigue } \\
\text { siendo mínimo, por lo } \\
\text { que no se recomienda la } \\
\text { profilaxis de TEV de } \\
\text { rutina en las guías } \\
\text { actuales. No se anotan } \\
\text { Intervalos de Confianza } \\
\text { y Significancia } \\
\text { Estadística }\end{array}$ & $\begin{array}{l}\text { Se necesita } \\
\text { más } \\
\text { investigación } \\
\text { para observar } \\
\text { la efectividad } \\
\text { potencial y } \\
\text { los perfiles de } \\
\text { seguridad a } \\
\text { largo plazo de } \\
\text { los nuevos } \\
\text { anticoagulant } \\
\text { es orales en la } \\
\text { madre y el } \\
\text { feto. Además, } \\
\text { existe la } \\
\text { necesidad de } \\
\text { ensayos } \\
\text { controlados } \\
\text { aleatorios } \\
\text { para } \\
\text { investigar } \\
\text { posibles } \\
\text { estrategias de } \\
\text { tratamiento } \\
\text { para aliviar } \\
\text { los síntomas } \\
\text { asociados con } \\
\text { las venas } \\
\text { varicosas y la } \\
\text { estasis } \\
\text { venosa. }\end{array}$ & & \\
\hline $\begin{array}{l}\text { Smyth } \\
2015\end{array}$ & $\begin{array}{l}\text { Evaluar } \\
\text { cualquier forma } \\
\text { de intervención } \\
\text { utilizada para } \\
\text { aliviar los } \\
\text { síntomas } \\
\text { asociados con } \\
\text { las venas } \\
\text { varicosas y el } \\
\text { edema de la } \\
\text { pierna en el } \\
\text { embarazo. } \\
\text { Periodo de } \\
\text { búsqueda: } \\
\text { No se reporta } \\
\text { fecha de inicio } \\
\text { de búsqueda, } \\
\text { pero finaliza el } \\
\text { 5/ } 2015 \text {. }\end{array}$ & $\begin{array}{l}\text { Mujeres } \\
\text { embarazada } \\
\text { s con venas } \\
\text { varicosas o } \\
\text { edema en } \\
\text { las piernas, } \\
\text { o ambos, en } \\
\text { cualquier } \\
\text { periodo de } \\
\text { gestación }\end{array}$ & $\begin{array}{l}\text { Cualquier } \\
\text { método descrito } \\
\text { utilizado para } \\
\text { tratar venas } \\
\text { varicosas o } \\
\text { edema de } \\
\text { piernas } \\
\text { comparado con } \\
\text { placebo o } \\
\text { ningún } \\
\text { tratamiento, o } \\
\text { cualquier otro } \\
\text { método. } \\
\text { Estos pueden } \\
\text { incluir: } \\
\text { 1. } \\
\text { Intervenciones } \\
\text { farmacológicas } \\
\text { (escleroterapia }\end{array}$ & $\begin{array}{l}\text { 1. Rutósidos vs } \\
\text { Placebo. } \\
\text { 2. Compresión } \\
\text { intermitente } \\
\text { neumática } \\
\text { externa vs } \\
\text { Reposo. } \\
\text { 3. Reflexología } \\
\text { vs Descanso. } \\
\text { 4. Rexología } \\
\text { linfática vs } \\
\text { Reflexología } \\
\text { relajante. } \\
\text { 5. Inmersión en } \\
\text { agua vs } \\
\text { Elevación de la } \\
\text { pierna. } \\
\text { 6. Masaje de } \\
\text { pies vs Cuidado } \\
\text { de rutina. }\end{array}$ & $\begin{array}{l}\text { Son } 6 \text { estudios: } \\
\text { 1. Rutosidos vs Placebo: } \\
69 \text { mujeres. } \\
\text { 2. Compresión } \\
\text { neumática externa vs } \\
\text { Descanso: } 35 \text {. } \\
\text { 3. Reflexología linfática } \\
\text { vs reflexología relajante: } \\
\text { 45. } \\
\text { 4. Reflexología vs } \\
\text { descanso: } 55 \text { mujeres. } \\
\text { 5. Inmersión en agua vs } \\
\text { elevación de las piernas: } \\
\text { 32 mujeres. }\end{array}$ & $\begin{array}{l}\text { 1. Los } \\
\text { rutósidos } \\
\text { parecen } \\
\text { ayudar a las } \\
\text { mujeres con } \\
\text { venas } \\
\text { varicosas que } \\
\text { experimentan } \\
\text { síntomas } \\
\text { (calambres } \\
\text { nocturnos, } \\
\text { sensación de } \\
\text { cansancio, } \\
\text { parestesia). } \\
\text { Sin embargo } \\
\text { la } \\
\text { investigación } \\
\text { no presentó } \\
\text { suficientes } \\
\text { datos para } \\
\text { evaluar la }\end{array}$ & $\begin{array}{l}\text { La investigación } \\
\text { realizó una } \\
\text { evaluación } \\
\text { minuciosa de } \\
\text { cada estudio y las } \\
\text { intervenciones } \\
\text { que se utilizaron. } \\
\text { La evidencia } \\
\text { encontrada } \\
\text { respecto a las } \\
\text { intervenciones de } \\
\text { venas varicosas } \\
\text { en el embarazo es } \\
\text { limitada y } \\
\text { antigua. } \\
\text { Se necesitan } \\
\text { estudios } \\
\text { controlados } \\
\text { aleatorizados con } \\
\text { poblaciones más }\end{array}$ & Alta \\
\hline
\end{tabular}




\begin{tabular}{|c|c|c|c|c|c|c|c|c|}
\hline Referencia & Estudio & Población & Intervención & Comparación & Resultados & Conclusión & Comentario & $\begin{array}{l}\text { Calidad de } \\
\text { la evidencia }\end{array}$ \\
\hline & $\begin{array}{l}\text { Diseño: } \\
\text { Estudios } \\
\text { Aleatorios de } \\
\text { tratamiento para } \\
\text { las venas } \\
\text { varicosas o } \\
\text { edema en las } \\
\text { piernas, o } \\
\text { ambos, en el } \\
\text { embarazo. }\end{array}$ & & $\begin{array}{l}\text { o flebotónicos) } \\
2 . \\
\text { Intervenciones } \\
\text { no } \\
\text { farmacológicas } \\
\text { (medias de } \\
\text { compresión, } \\
\text { elevación de la } \\
\text { pierna, } \\
\text { cualquier forma } \\
\text { de descanso, } \\
\text { cualquier forma } \\
\text { de ejercicio, } \\
\text { inmersión en } \\
\text { agua, } \\
\text { reflexología, } \\
\text { fisioterapia, } \\
\text { masaje). } \\
\text { 3. } \\
\text { Intervenciones } \\
\text { quirúrgicas. }\end{array}$ & & 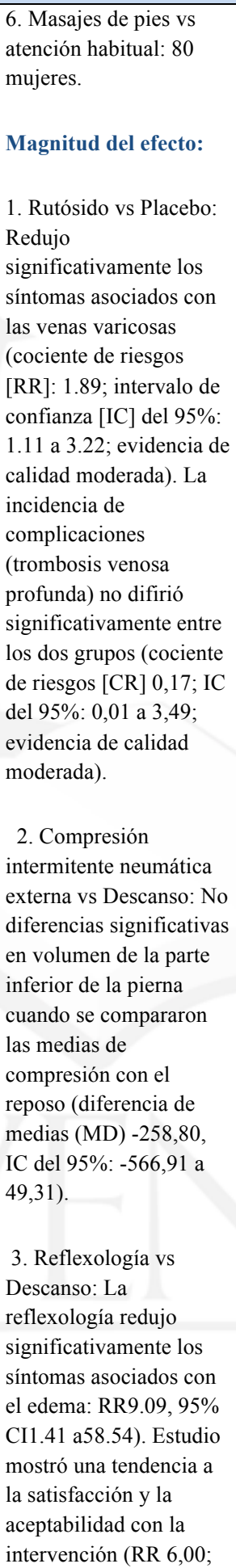 & 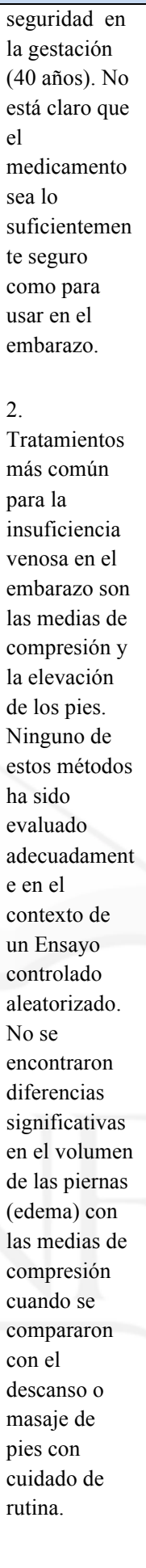 & $\begin{array}{l}\text { grandes que } \\
\text { permitan dar un } \\
\text { sustento a una } \\
\text { adecuada } \\
\text { intervención. }\end{array}$ & \\
\hline
\end{tabular}




\begin{tabular}{|c|c|c|c|c|c|c|c|c|}
\hline Referencia & Estudio & Población & Intervención & Comparación & Resultados & Conclusión & Comentario & $\begin{array}{l}\text { Calidad de } \\
\text { la evidencia }\end{array}$ \\
\hline & & & & & $\begin{array}{l}\text { IC del } 95 \% \text { : } 0,92 \text { a } \\
\text { 39,11). } \\
\text { 4. Inmersión en agua vs } \\
\text { Elevación de la pierna: } \\
\text { Inmersión en agua } \\
\text { durante } 20 \text { minutos en } \\
\text { una piscina reduce el } \\
\text { volumen de la pierna } \\
\text { (RR 0,43; IC del 95\%: } \\
\text { 0,22 a 0,83). } \\
\text { 5. Masaje de pies vs } \\
\text { Cuidado de rutina: No } \\
\text { diferencia significativa } \\
\text { en el perímetro de la } \\
\text { pierna cuando se } \\
\text { comparó el masaje de } \\
\text { pies con la atención de } \\
\text { rutina (DM - } 0,11, \text { IC del } \\
\text { 95\%: - } 1,02 \text { a } 0,80) .\end{array}$ & $\begin{array}{l}\text { 3. La } \\
\text { seguridad de } \\
\text { las } \\
\text { intervencione } \\
\text { s tanto } \\
\text { farmacológica } \\
\text { s como no } \\
\text { farmacológica } \\
\text { s en esta } \\
\text { revisión no se } \\
\text { pueden } \\
\text { verificar a } \\
\text { partir de los } \\
\text { datos } \\
\text { disponibles. } \\
\text { 4. No se } \\
\text { puede } \\
\text { proporcionar } \\
\text { una guía clara } \\
\text { con respecto a } \\
\text { cualquier } \\
\text { forma de } \\
\text { intervención } \\
\text { para restaurar } \\
\text { los síntomas } \\
\text { asociados con } \\
\text { las venas } \\
\text { varicosas y el } \\
\text { edema en el } \\
\text { embarazo. Se } \\
\text { ha } \\
\text { identificado } \\
\text { que es } \\
\text { necesario la } \\
\text { realización de } \\
\text { ensayos } \\
\text { controlados } \\
\text { aleatorios } \\
\text { grandes, bien } \\
\text { diseñados que } \\
\text { permitan } \\
\text { extraer } \\
\text { conclusiones } \\
\text { más robustas. } \\
\text { 5. La } \\
\text { evidencia } \\
\text { disponible es } \\
\text { insuficiente } \\
\text { para extraer } \\
\text { conclusiones }\end{array}$ & & \\
\hline
\end{tabular}




\begin{tabular}{|l|l|l|l|l|l|l|l|l|}
\hline Referencia & Estudio & Población & Intervención & Comparación & Resultados & Conclusión & Comentario & $\begin{array}{c}\text { Calidad de } \\
\text { la evidencia }\end{array}$ \\
\hline & & & & & & $\begin{array}{l}\text { confiables } \\
\text { para la } \\
\text { práctica } \\
\text { clínica. }\end{array}$ & \\
\hline
\end{tabular}

Fuente: Elaboración propia.

Tabla 5. AGREE II. Varicose veins in the legs. The diagnosis and management of varicose veins. Clinical Guideline Methods, 2013.

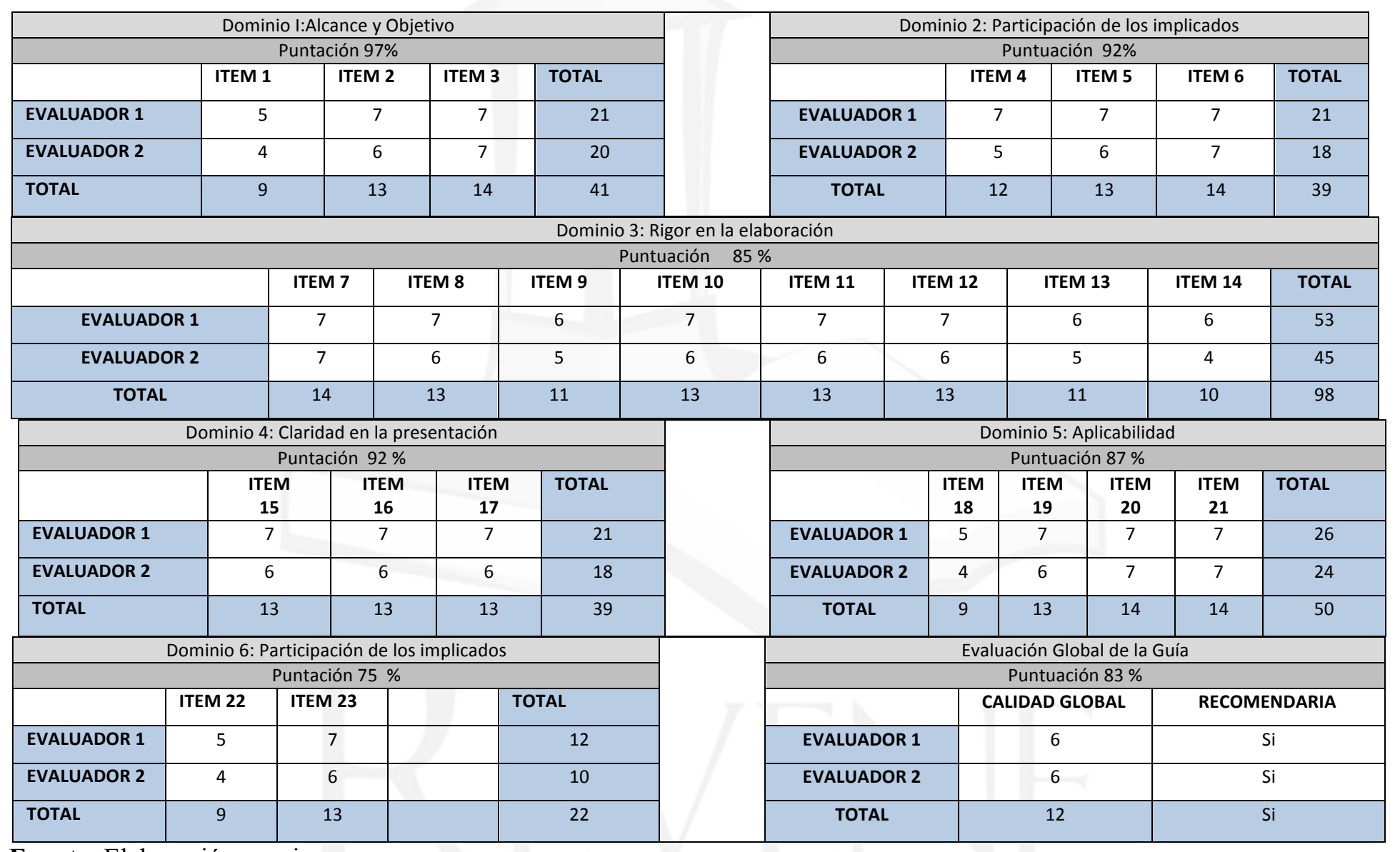

Fuente: Elaboración propia 


\section{DISCUSION}

Según Taylor et al. ${ }^{24}$ en su artículo titulado: “The Hemodinamyc effects of pregnancy on the lower extremity" la insuficiencia venosa crónica es resultado de anomalías estructurales y bioquímicas de la pared de la vena. Los autores señalan que el manejo de dicha patología se divide en tres grupos principales que son: tratamientos farmacológicos, tratamientos no farmacológicos y los tratamientos quirúrgicos. Cuando se presenta este tipo de patología el edema es una condición que puede estar presente, estableciendo como objetivo principal la reducción de los síntomas y la utilización de enfoques farmacológicos y no farmacológicos ${ }^{25}$.

De igual forma Smyth et al. ${ }^{11}$ afirma que los métodos para tratar las venas varicosas o el edema en miembros inferiores en la mujer gestante incluye intervenciones farmacológicas tales como los flebotónicos o la escleroterapia, intervenciones no farmacológicas como el uso de medias de compresión, elevación de las piernas, descanso, ejercicio, inmersión en agua y reflexología; por último, como tercera opción señala las intervenciones quirúrgicas.

Otro autor también menciona que los flebotónicos son tratamientos farmacológicos que funcionan a través del aumento del tono venoso y la prevención del edema ${ }^{26}$. A pesar que estos fármacos poseen mecanismos de acción venotónicos, la mayoría de los estudios muestran una evidencia de beneficio de calidad baja. En una revisión bibliográfica realizada en Cochrane solo el $28 \%$ de los estudios incluidos sobre este tema incluyeron criterios diagnósticos estándar.

Sobre lo anterior Smith et al. ${ }^{11}$ en su estudio define los flebotónicos como fármacos venoactivos que se asocian con el aumento del tono venoso y la prevención del edema.

Dichos fármacos incluyen rutósidos, hidrosmina, diosmina, dobesilato de calcio, cromocarbono, centella asiática, floberdato disódico, extracto de corteza de pino marítimo francés, extracto de semilla de uva, aminaftone y el 0(b-hydroxythylrutoside) que es un compuesto semisintético. Aunque los rutósidos pueden ayudar en el manejo de las venas varicosas aún se desconoce si el medicamento es seguro durante el embarazo.

Dentro de este contexto, Bergstein ${ }^{27}$ realizó un ECA que comparó la eficacia del flebotónico oral O -(bhidroxietil) rutósido, con el placebo para el tratamiento de las venas varicosas durante el embarazo. En este estudio se demostró que el rutósido redujo significativamente la sintomatología asociada con las venas varicosas con un RR de 1.89 y un IC del 95\% 1.11 a 3.22. En este estudio no hubo diferencias estadísticas significativas en la tasa de efectos secundarios entre los grupos de tratamiento.

Sobre estos fármacos Smyth et al. ${ }^{11}$ también refiere que los rutósidos son la intervención más efectiva para reducir los síntomas de las venas varicosas durante el embarazo.

Esto lo reafirman Rodríguez et al. ${ }^{28}$ en el 2017 en su investigación titulada “Actualización del tratamiento de la insuficiencia venosa en la gestación" cuando exponen que existe evidencia de calidad moderada que sugiere que los rutósidos parecen ayudar a aliviar los síntomas de las venas varicosas al final del embarazo y que estos fármacos tienen un efecto beneficioso a la hora de tratar la insuficiencia venosa crónica en el tercer trimestre del embarazo. 
En diferente perspectiva se analizan otros tipos de tratamientos invasivos, específicamente las terapias de ablación venosa, la escleroterapia y los tratamientos quirúrgicos para el manejo de la patología varicosa en gestantes. Sobre este tema Rodríguez et al. ${ }^{28}$ plantean que las terapias de ablación venosa generalmente se encuentran contraindicadas en el embarazo y que deben esperarse al menos dos meses tras el mismo para plantear las opciones quirúrgicas puesto que gran parte de las manifestaciones de la enfermedad venosa de la gestante revierten durante este periodo.

Algunos investigadores han planteado que la cirugía radical, la escleroterapia o una combinación de ambas son el tratamiento definitivo para las venas varicosas durante el embarazo. Sin embargo Greenstone et al. ${ }^{29}$ mencionan que el uso de esclerosantes podría aumentar el riesgo de sufrir de tromboflebitis ascendente no controlada. Estos mismos autores refieren que la incidencia y recurrencia es mucho mayor cuando se realizan las cirugías radicales durante el embarazo, debido al aumento de la presión venosa, a la obstrucción del drenaje venoso de las extremidades inferiores y a los factores hormonales.

De igual manera Smyth et al. ${ }^{11}$ señala que el tratamiento quirúrgico de las varices está contraindicado en el embarazo y ha de esperarse al menos dos meses tras el parto para valorar su indicación debido al gran número de manifestaciones de la insuficiencia venosa crónica que revierten espontáneamente durante este periodo. Así mismo lo plantea Cleave et al. ${ }^{30}$ en su artículo titulado "Varicose Veins and Pregnancy" al afirmar que el tratamiento activo durante el embarazo se reserva para las varices graves con incompetencia valvular que no responden al tratamiento conservador.

Solanki et al. ${ }^{31}$ en la Guía Clínica titulada: "Varicose veins in the legs. The diagnosis and management of varicose veins", consideran que las ventajas clínicas del tratamiento invasivo por varices durante el embarazo no pesaron más que los daños posibles a la mujer y al feto. Afirman en esta investigación que la preocupación primaria es la seguridad para el binomio madre-hijo y que el tratamiento no se aconseja en mujeres embarazadas. Pese a esto, los investigadores señalan que podría haber algunas situaciones excepcionales, donde la intervención se podría considerar.

Sobre esto existe un consenso general que ese tiempo debería de ser de 3 a 6 meses debido a la normalización del cuerpo después del parto y para disminuir el riesgo de introducir fármacos al lactante durante el amamantamiento $^{31}$.

Por otro lado, Taylor et al. ${ }^{24}$ señala que el enfoque del tratamiento no farmacológico es disminuir al máximo la necesidad del uso de medidas invasivas. Refiere que los métodos no farmacológicos para tratar la insuficiencia venosa en la mujer embarazada incluyen el uso de medias de compresión, descanso, elevación de las piernas, reflexología, inmersión en agua y masajes. Además, considera que algunos tratamientos conservadores como la reflexología o la inmersión en agua parecen ayudar a mejorar los síntomas en mujeres con edema de piernas, pero una vez más esto se basa en dos estudios pequeños de 43 y 32 mujeres, respectivamente. En estos estudios se descubrió que tanto la reflexología como la inmersión en agua reducen los síntomas del edema de la pierna; sin embargo, estos hallazgos se basaron en estudios únicos que no se han reproducido. 
Por otro lado, se menciona que las intervenciones no farmacológicas que incluyen el uso de vendajes, medias de compresión y la elevación de las piernas pueden aliviar la incomodidad y mejorar los síntomas en algunas mujeres, sin embargo, falta evidencia para apoyar estas terapias ${ }^{26}$.

Algunos de estos estudios han demostrado que la reflexología puede reducir más los síntomas de la IVC en comparación con el reposo y la inmersión en agua, ya que es una terapia antigua natural mediante la cual se aplica una presión del dedo y el pulgar a los puntos de flexión en los pies, las manos y los oídos, con el fin de reordenar ciertas áreas en el cuerpo. La reflexología linfática es una versión de esta técnica que imita el drenaje linfático, ayudando a mover el fluido extravascular hacia el espacio intravascular en los miembros inferiores edematosos. El mismo efecto produce la inmersión en agua solo que utiliza la presión hidrostática ejercida uniformemente en las piernas $^{24}$.

Otros autores como Nesbitt et al. ${ }^{32}$ en el 2014, afirman que las técnicas más utilizadas en el tratamiento general de venas varicosas son el tratamiento conservador también conocido como terapia de compresión, o tratamientos intervencionistas, pero que las pacientes gestantes con venas varicosas deben ser tratadas idealmente con terapia de compresión, escleroterapia y flebectomía.

Jamieson, Calderwood y Greer $^{33}$ en su investigación evaluó el uso de medias de compresión en el postparto inmediato y encontró una reducción en el diámetro de la vena femoral común con un aumento de la velocidad de la sangre venosa después de 30 minutos de terapia de compresión, lo que nos indica que el tratamiento compresivo es exitoso en la disminución de la estasis venoso en el postparto y disminuir el riesgo de IVC. Igualmente, Smyth et al. ${ }^{11}$ menciona que las medias de compresión pueden aliviar la hinchazón y el dolor de piernas, evitando que se desarrollen más venas varicosas. Afirman que deben usarse durante el día y quitarse por la noche, que las medias de compresión graduadas deben usarse a lo largo de la pierna para favorecer de mejor manera el retorno venoso hacia el corazón.

Dentro de este contexto, Smyth et al. ${ }^{11}$ reafirma que los tratamientos más comunes para la insuficiencia venosa en el embarazo son las medias de compresión y la elevación de los pies; pero que ninguno de estos métodos ha sido evaluado adecuadamente en el contexto de un ensayo controlado aleatorizado.

Greenstone et al. ${ }^{29}$ es otro autor que apoya el pensamiento de que la gestión básica de todos los grados de varicosis durante el embarazo debe ser por medios conservadores, tales como un soporte elástico adecuado que posean la suficiente resistencia para mantener las varices completamente comprimidas. Así mismo Taylor et al. ${ }^{24}$ y Solanki et al. ${ }^{31}$ consideran que la terapia de compresión es ventajosa ya que es económica y fácil de colocar, pero que su principal limitación es el incumplimiento del usuario, lo que limita su efectividad.

Al igual que los autores anteriores Carrasco $^{34}$ considera que las medias de compresión durante el embarazo no previenen la aparición de varices, pero pueden disminuir la incidencia de insuficiencia venosa y mejorar la sintomatología. Así mismo Rodríguez et al. ${ }^{28}$ en su investigación señalan que la correcta utilización de este método ha demostrado ser el tratamiento más eficaz de la insuficiencia venosa crónica, ya que su mecanismo de acción es mejorar el retorno venoso al reducir la presión venosa y el reflujo. Además retrasan la progresión de la enfermedad. 
Pese a lo expuesto anteriormente, Thaler et al. ${ }^{2}$ en un ensayo demostró que el uso de medias de compresión profiláctica no previno la aparición de venas varicosas pero si redujo significativamente la incidencia del reflujo de la vena safena en la unión safeno femoral, y mejoro los síntomas de las piernas. Del mismo modo Solanki et al. ${ }^{31}$ señala que las mejoras de la calidad de vida de la terapia de compresión probablemente justificarán el coste adicional; por lo tanto se considera que este método es rentable para mujeres durante el embarazo.

Otro argumento relevante a lo largo de esta investigación es el expuesto por Taylor et al. ${ }^{24}$ que afirma que existe la necesidad de ensayos controlados aleatorios para investigar posibles estrategias de tratamiento para aliviar los síntomas de las venas varicosas y la estasis venosa. El autor señala que existen estrategias para manejar la enfermedad venosa de las extremidades inferiores asociada con el embarazo. Pero que lastimosamente la calidad y el alcance de las investigaciones científicas basadas en el manejo de las varices en estadio temprano durante el embarazo son modestos.

Por otro lado, el grupo de investigadores que dirigió la realización de la Guía Clínica de Solanki et al. ${ }^{31}$ concluyó que los beneficios clínicos del tratamiento de las venas varicosas con medias de compresión durante el embarazo pueden ser mayores que los posibles daños para la madre o el feto. Consideran que las terapias intervencionistas están contraindicadas en el embarazo por lo que señalan la terapia de compresión como la única opción viable que ha demostrado incluso una mejora en la calidad de vida de las usuarias; sin embargo, ellos están de acuerdo como muchos autores en la necesidad de estudios mayores.

\section{CONCLUSION}

Las medias de compresión es considerado el tratamiento más indicado para el alivio y reducción de la sintomatología asociados con las venas varicosas en el embarazo; sin embargo, no previene la aparición posterior. El tratamiento compresivo no ha sido evaluado de forma adecuada bajo un ensayo controlado aleatorio riguroso y confiable que permita establecer una pauta clara. Del mismo modo, los rutósidos y los anticoagulantes orales parecen ayudar a las mujeres con venas varicosas en el embarazo; sin embargo, según la evidencia científica no queda claro que dichos medicamentos sea lo suficientemente seguros en la gestación tanto para la madre como para el feto.

El tratamiento invasivo incluyendo el quirúrgico no es recomendable durante el embarazo debido a que los riesgos superan los beneficios. Se sugiere que este tipo de intervenciones no se realicen durante la gestación solo en casos delicados que lo ameriten. La literatura sugiere que el tiempo adecuado para el tratamiento invasivo incluyendo el quirúrgico es cuando la usuaria considere que no desea tener más hijos, o unos meses después del nacimiento del bebé.

La reflexología, masajes en los pies e inmersión en agua parecen ayudar a reducir el edema en miembros inferiores en mujeres embarazadas.

\section{Declaración de conflicto de intereses.}

Las investigadoras declaran que en la presente investigación no tienen ningún conflicto de intereses de índole personal, social, económico ni laboral. 


\section{REFERENCIAS BIBLIOGRÁFICAS}

1.Cossart, L. Varicose veins and pregnancy. Br J Surg. 2001 Dec; 88:323-4. DOI: https://doi.org/10.1046/j.1365-2168.2001.01708.x

2. Thaler E, Huch R, Huch A, Zimmermann R. Compression stockings prophylaxis of emergent varicose veins in pregnancy: a prospective randomised controlled study. Swiss Med Wkly. 2001 Dec 1; 131(45-46). DOI: www.2001/45/smw-09805

3. Bamigboye A, Hofmeyr G. Interventions for leg edema and varicosities in pregnancy. What evidence?. Eur J Gynecol Reprod Biol. 2006 Nov; 129(1): 3-8. DOI: www.10.1016/j.ejogrb.2006.03.008

4. Lenković M, Cabrijan L, Gruber F, Batinac T, Manestar-Blazić T, Stanić Zgombić Z, Stasić A. Effect of Progesterone and Pregnancy on the Development of Varicose Veins. Acta Dermatovenerol Croat. 2009; 17(4):263-7. https://www.ncbi.nlm.nih.gov/pubmed/20021978

5. Sell H, Vikatmaa P, Albäck A, Lepäntalo M, Malmivaara A.; Mahmoud O, Venermo M. Compression Therapy Versus Surgery in the Treatment of Patient with Varicose Veins: A RCT. Eur J Vasc Endovasc Surg. 2014 Jun; 47(6): 670-7. DOI: www.10.1016/j.ejvs.2014.02.015

6. Lohr JM, Bush R. Venous disease in women: Epidemiology, manifestations, and treatment. J Vasc Surg. 2013 Apr; 57(4): 37S-45S. DOI: https://doi.org/10.1016/j.jvs.2012.10.121

7. Vandy F, Wakefield TW. Varicose veins: evaluating modern treatments, with emphasis on powered phlebectomy for branch varicosities. Interventional Cardiology. 2012 Oct; 4(5): 527-536. DOI: www.10.2217/ica.12.48

8. Zhang S, Melander S. Varicose Veins: Diagnosis, Management and Treatment. J Nurse Pract. 2014 Jun; 10(6):417-424. DOI: https://doi.org/10.1016/j.nurpra.2014.03.004

9. Jundt J, Liem T, Moneta G. Venous and Lymphatic Disease [Internet]. En: Brunicardi F, Andersen D, Billiar T, et al, editores. Schwartz's Principles of Surgery. 10ma ed. USA: McGraw-Hill Education; 2015. http://accessmedicine.mhmedical.com

10. Winterborn R, Smith F. Varicose Veins. Surg J. 2010 Jun; 28(6):259-262. DOI: https://doi.org/10.1016/j.mpsur.2010.01.011

11. Smyth R, Aflaifel N, Bamigboye AA. Interventions for varicose veins and leg oedema in pregnancy (Review). Cochrane Database of Systematic Reviews. 2015; 10. DOI: www.10.1002/14651858.CD001066.pub3 
12. De Barros N, Janeiro MC, de Amorim J, Miranda F. Pregnancy and lower limb varicose veins: prevalence and risk factors. J Vasc Bras. 2010 Jun; 9(2):29-35. DOI:

http://dx.doi.org/10.1590/S1677-54492010000200004

13. Ombrellino M, Kabnick L. Varicose Vein Surgery. Semin Intervent Radiol. 2005 Sep; 22(3):185194. DOI: www.10.1055/s-2005-921951

14. Kawata E, Kondoh E, Kawasaki K, Baba T, Ueda A, Kido A, Konishi, I. Utero-ovarian varices and absent inferior vena cava in pregnancy. J Obstet Gynaecol Res. 2015 Apr; 41(4), 631-4. DOI: www.10.1111/jog.12599

15. Vargas, Z. La investigación aplicada: una forma de conocer las realidades con evidencia científica. Revista Educación. 2009; 33(1):155-165. https://revistas.ucr.ac.cr/index.php/educacion/article/viewFile/538/589

16. Murillo, W. La investigación científica. Monografías.com; 2008. http//www.monografias.com/trabajos15/invest-científica/investcientífica.shtm

17. Coello P, Ezquerro O, Fargues I, García J, Marzo M, Navarra M, Pardo J, Subirana M, Urrutia G. Enfermería Basada de la Evidencia. Hacia la excelencia de los cuidados. Madrid: Difusión Avances de Enfermería.; 2004. https://ebevidencia.com/wp-content/uploads/2013/08/EBE.-Haciala-excelencia-en-cuidados.pdf

18. Soberats S,Vergara E. Acerca de la práctica clínica basada en la evidencia.Rev Cubana Med Gen Integr. 1999 Jul-Ago; 15(4):355-356. http://scielo.sld.cu/scielo.php?script=sci_arttext\&pid=S086421251999000400001

19. González J, Buñuel A. Búsqueda eficiente de las mejores pruebas científicas disponibles en la literatura: fuentes de información primaria y secundaria. Evid Pediatr. 2006 Feb; 2(12): 1-10. https://evidenciasenpediatria.es/articulo/5026/busqueda-eficiente-de-las-mejores-pruebascientificas-disponibles-en-la-literatura-fuentes-de-informacion-primarias-y-secundarias

20. Flórez I, Montoya D. Las guías de práctica clínica y el instrumento Agree II. Rev Colomb Psiquiatr. 2011 Jul -Sep; 40(3):563-576. https://www.elsevier.es/es-revista-revista-colombianapsiquiatria-379-avance-resumen-las-guias-practica-clinica-elS0034745014601475.DOI:10.1016/S0034-7450(14)60147-5

21. Sackett D1, Rosenberg WMC, Gary JAM, Haynes RB, Richardson WS. Evidence based medicine: what is it and what it isn't. BMJ 1996; 312:71-2. DOI: www.10.1136/bmj.312.7023.71

22. Alcolea M, Oter C, García A. Fases de la Práctica Basada en la Evidencia. Nure Investigación. 2011 Jul-Ago; (53): 1-5. http://www.nureinvestigacion.es/OJS/index.php/nure/article/view/545/534 
23. Laguna S, Caballero C, Lewis V, Mazuera1 S, Salamanca J, Daza1 W, Fourzali A. Consideraciones éticas en la publicación de investigaciones científicas. Sal Uninor [Internet]. 2007; 23(1):64-78. http://www.scielo.org.co/pdf/sun/v23n1/v23n1a08

24. Taylor J, Hicks C, Heller J. The hemodynamic effects of pregnancy on the lower extremity venous system. J Vasc Surg Venous Lymphat Disrd. 2018 Mar; 6(2):246-255. http://dx.doi.org/10.1016/j.jvsv.2017.08.001

25. Campbell B. Varicose veins and their management.BMJ .[Internet].2006; 333(7562):287-92. DOI: www.10.1136/bmj.333.7562.287

26. Bartholomew J, King T, Sahgal A, Vidimos AT. Varicose veins: newer, better treatments available. Cleveland Clinic Journal of Medicine,2005;72(4):312-4, 319-21, 325-8. DOI: www.10.3949/ccjm.72.4.312

27. Bergstein N. Clinical study on the efficacy of O-(BetaHydroxyethyl) Rutoside (HR) in varicosis of pregnancy. Journal of International Medical Research [internet]. 1975; 3:189-93. DOI: https://doi.org/10.1177/030006057500300308

28. Rodríguez N, Álvarez E. Actualización del tratamiento de la insuficiencia venosa en la gestación. Semergen. 2017. https://doi.org/10.1016/j.semergen.2017.11.003

29. Greenstone M, Craig E, Massell T. Management of Varicose Veins During Pregnancy.Calif Med.1957 Dec; 87(6): 365-7. https://www.ncbi.nlm.nih.gov/pmc/articles/PMC1512200/

30. Cleave T. Varicose Veins and Pregnancy. Br Med J. 1965; (2): 1183. https://doi.org/10.1136/bmj.2.5471.1183-C

31. Solanki J, Fenu E, Flynn N, Ritchie G. Varicose veins in the legs. The diagnosis and management of varicose veins. National Clinical Guideline Centre. 2013 Jul. https://www.ncbi.nlm.nih.gov/pubmed/25535637

32. Nesbitt, C; Bedenis, R; Bhattacharya, V, Satansby G. Endovenous ablation (radiofrequency and laser) and foam sclerotherapy versus open surgery for great saphenous vein varices. Cochrane Database of Systematic Reviews. 2014; (7). http://onlinelibrary.wiley.com/doi/10.1002/14651858.CD005624.pub3/pdf

33. Jamieson R, Calderwood C, Greer I. The effect of graduated compression stockings on blood velocity in the deep venous system of the lower limb in the postnatal period. BJOG.2007;114:1292-4. DOI: www.10.1111/j.1471-0528.2007.01421.x

34. Carrasco E, Díaz S. Recomendaciones para el manejo de la enfermedad Venosa crónica en atención primaria. ID Médica. 2015:12-6. https://www.semergen.es/resources/files/noticias/venosaCrocina_1.pdf 